\title{
A Necessary and Sufficient Condition for Generalized Demixing
}

\author{
Chun-Yen Kuo, Gang-Xuan Lin, and Chun-Shien Lu
}

\begin{abstract}
Demixing is the problem of identifying multiple structured signals from a superimposed observation. This work analyzes a general framework, based on convex optimization, for solving demixing problems. We present a new solution to determine whether or not a specific convex optimization problem built for generalized demixing is successful. This solution also creates a way to estimate the probability of success by the approximate kinematic formula.
\end{abstract}

Index Terms-Compressive sensing, conic geometry, convex optimization, $\ell_{1}$-minimization, sparse signal recovery.

\section{INTRODUCTION}

A CCORDING to the theory of convex analysis, convex cones have been exploited to express the optimal conditions for a convex optimization problem [6]. In particular, Amelunxen et al. [1] presented the necessary and sufficient conditions for the problems of basis pursuit (BP) and demixing to be successful.

Let $x_{0} \in \mathbb{R}^{n}$ be an unknown $k$-sparse vector with $k$ nonzero entries in a certain domain, let $A$ be an $m \times n$ random matrix whose entries are independent standard normal variables, and let $z=A x_{0} \in \mathbb{R}^{m}$ be the measurement vector obtained via random transformation by $A$. In regard to the basis pursuit (BP) problem, which is defined as

$$
\text { (BP) }\left\{\text { minimize } \quad\|x\|_{1} \quad \text { subject to } \quad z=A x,\right.
$$

a convex optimization method was proposed in Chen et al. [4] to solve the sparse signal recovery problem in the context of compressive sensing [5] when $m<n$.

To explore whether BP has a unique optimal solution, Amelunxen et al. [1] started from the concept of the conic integral.

Definition 1.1. (descent cone): [1] The descent cone $\mathcal{D}(f, x)$ of a proper convex function $f: \mathbb{R}^{n} \rightarrow \overline{\mathbb{R}}$ at the point $x \in \mathbb{R}^{n}$ is the conical hull of the perturbations that do not increase $f$ near $x$.

$$
\mathcal{D}(f, x):=\bigcup_{\tau>0}\left\{y \in \mathbb{R}^{n}: f(x+\tau y) \leq f(x)\right\} .
$$

Manuscript received March 22, 2015; revised June 01, 2015; accepted July 13, 2015. Date of publication July 16, 2015; date of current version July 21 , 2015. This work was supported by MOST Grants 102-2221-E-001-002-MY2 and 102-2221-E-001-022-MY2. The associate editor coordinating the review of this manuscript and approving it for publication was Prof. Negar Kiyavash.

The authors are with Institute of Information Science, Academia Sinica, Taipei, Taiwan, R.O.C. (e-mail: r00221004@ntu.edu.tw; spybeiman@gmail. com; 1cs@iis.sinica.edu.tw).

Color versions of one or more of the figures in this paper are available online at http://ieeexplore.ieee.org.

Digital Object Identifier 10.1109/LSP.2015.2457403
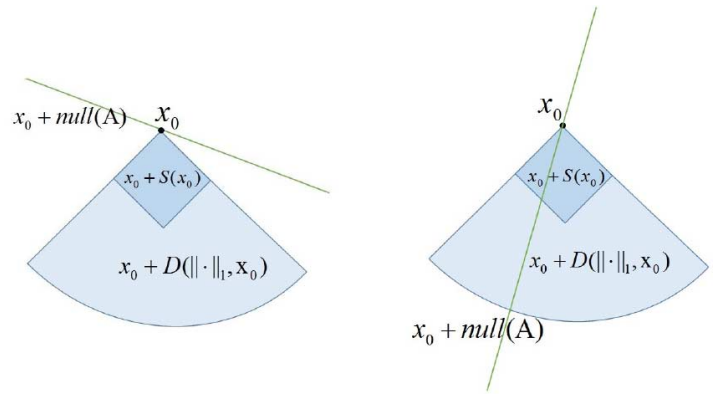

Fig. 1. The optimality condition for the BP problem. [Left] BP succeeds. [Right] BP fails. $S\left(x_{0}\right)=\left\{y \in \mathbb{R}^{d}:\left\|x_{0}+y\right\|_{1} \leq\left\|x_{0}\right\|_{1}\right\}$.

We say that problem BP, defined in Eq. (I.1), succeeds when it has a unique minimizer $\hat{x}$ that coincides with the true unknown, that is, $\hat{x}=x_{0}$. To characterize when the BP problem succeeds, Amelunxen et al. presented the primal optimality condition as:

$$
\operatorname{null}(A) \cap \mathcal{D}\left(\|\cdot\|_{1}, x_{0}\right)=\{0\}
$$

in terms of the descent cone [1] ( $c f .$, [3] and [7]), where $\operatorname{null}(A)$ denotes null space of $A$. The optimality condition for the BP problem is also illustrated in Fig. 1.

Amelunxen et al. [1] also explored the demixing problem (sparse + sparse) characterized as

$$
z=x_{0}+U y_{0},
$$

where $U \in \mathbb{R}^{n \times n}$ is a known orthogonal matrix, $x_{0}$ is itself sparse, and $y_{0}$ is sparse with respect to $U$. The optimization problem of recovering signals $x_{0}$ and $y_{0}$ is formally defined as follows, which we call the demixing problem (DP) in short:

$$
\text { (DP) }\left\{\begin{array}{c}
\text { minimize }\|x\|_{1} \\
\text { subject to }\|y\|_{1} \leq\left\|y_{0}\right\|_{1} \text { and } z=x+U y .
\end{array}\right.
$$

They propose the primal optimality condition (also illustrated in Fig. 2) as

$$
\mathcal{D}\left(\|\cdot\|_{1}, x_{0}\right) \cap-U \mathcal{D}\left(\|\cdot\|_{1}, y_{0}\right)=\{0\}
$$

to characterize whether $\left(x_{0}, y_{0}\right)$ is the unique minimizer to problem (DP).

The authors in [1] also estimated the probabilities of success of problem (BP) and problem (DP) with Gaussian random sensing matrices by the approximate kinematic formula. They derived the probability ${ }^{1}$ using convex (descent) cones. Note

${ }^{1}$ Nevertheless, the authors still fail to calculate the actual probabilities. In fact, they only derive the bounds of probabilities that involve the calculation of statistical dimension. Unfortunately, up to now, the statistical dimension still cannot be calculated accurately. 


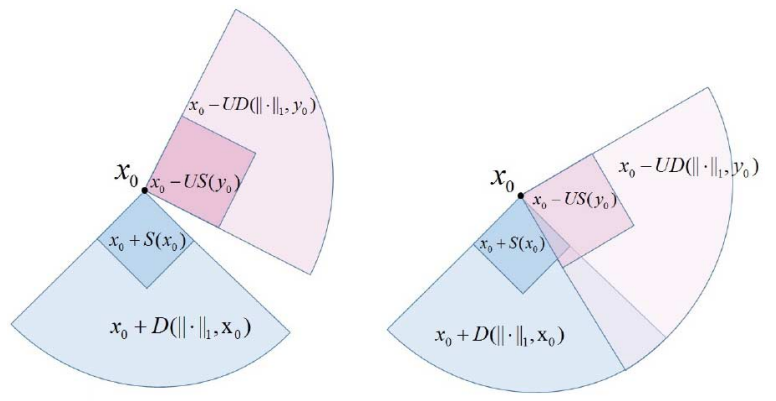

Fig. 2. The optimality condition for (DP) problem. [Left] problem (DP) succeeds. [Right] problem (DP) fails.

that, as shown in Fig. 1 and Fig. 2, the affine $\ell_{1}$ balls $S(\cdot)$ are defined as $S(a)=\left\{x:\|x+a\|_{1} \leq\|a\|_{1}\right\}$. Also note that cone $(S(a))=\mathcal{D}\left(\|\cdot\|_{1}, a\right)$, where cone $(S(a))$ is a conical hull of $S(a)$.

In Sections II and III, we generalize the demixing problem specified in Eq. (I.4), set the corresponding optimization problem to recover the signals of such generalized demixing model, and explore its necessary and sufficient condition for successful demixing.

\section{Motivation And Problem Definition}

The demixing problem we discuss in this paper refers to the extraction of two informative signals from a single observation. We consider a more general model for a mixed observation $z$ $\in \mathbb{R}^{m}$, which takes the form

$$
z=A x_{0}+B y_{0},
$$

where $x_{0} \in \mathbb{R}^{n_{1}}$ and $y_{0} \in \mathbb{R}^{n_{2}}$ are the unknown informative signals that we wish to find and where the matrices $A \in \mathbb{R}^{m \times n_{1}}$ and $B \in \mathbb{R}^{m \times n_{2}}$ are arbitrary linear operators (not necessarily $m \leq n_{1}$ or $n_{2}$ ). We assume that all elements appearing in Eq. (II.1) are known, except for $x_{0}$ and $y_{0}$. The broad applications of the general model in Eq. (II.1) can be found in [8] (and the references therein).

It should be noted that: (1) if $y_{0}$ in Eq. (II.1) is set to zero, then the generalized demixing model is degenerated to BP; (2) the demixing model in [1] is a special case of Eq. (II.1) if $A$ is set to an identity matrix and $B$ is enforced to be an orthogonal matrix; (3) our generalized demixing model has more freedom in the sense of dimension than that in [2] because $A$ and $B$ can be arbitrarily selected. Moreover, the two components $x_{0}$ and $y_{0}$ in our generalized model are permitted to have different lengths.

\section{MAIN RESULT}

The ground truths, $x_{0}$ and $y_{0}$, in Eq. (II.1) are approximated by solving the convex optimization problem, which we call the generalized demixing problem (GDP):

$$
\text { (GDP) }\left\{\begin{array}{c}
\text { minimize }\|x\|_{1} \\
\text { subject to }\|y\|_{1} \leq\left\|y_{0}\right\|_{1} \text { and } z=A x+B y .
\end{array}\right.
$$

We say problem (GDP) succeeds provided $\left(x_{0}, y_{0}\right)$ is the unique optimal solution to GDP. Our goal in this paper is to characterize when the problem (GDP) succeeds.
Theorem III.1: The problem (GDP) has a unique minimizer $(\hat{x}, \hat{y})$ to coincide with $\left(x_{0}, y_{0}\right)$ if and only if

$$
\left\{\begin{array}{c}
\operatorname{null}(A) \cap S\left(x_{0}\right)=\{0\}, \\
\operatorname{null}(B) \cap S\left(y_{0}\right)=\{0\}, \\
-A S\left(x_{0}\right) \cap B S\left(y_{0}\right)=\{0\} .
\end{array}\right.
$$

Proof: First, we assume that the problem (GDP) succeeds in having a unique minimizer $(\hat{x}, \hat{y})$ to coincide with $\left(x_{0}, y_{0}\right)$.

1) Claim: $\operatorname{null}(A) \cap S\left(x_{0}\right)=\{0\}$.

Given $h_{1} \in \operatorname{null}(A) \cap S\left(x_{0}\right)$, we have $A h_{1}=0$. By letting $\left(x^{\prime}, y^{\prime}\right)=\left(x_{0}+h_{1}, y_{0}\right)$, it follows that $z=A x^{\prime}+B y^{\prime}$ and $\left\|y^{\prime}\right\|_{1} \leq\left\|y_{0}\right\|_{1}$, which means that the point $\left(x^{\prime}, y^{\prime}\right)$ is a feasible point of problem (GDP). On the other hand, since $h_{1} \in S\left(x_{0}\right)$, we have $\left\|x_{0}+h_{1}\right\|_{1}=\left\|x^{\prime}\right\|_{1} \leq\left\|x_{0}\right\|_{1}$. By the fact that the problem (GDP) is assumed to have a unique minimizer $\left(x_{0}, y_{0}\right)$, we conclude that $h_{1}=0$.

2) Claim: $\operatorname{null}(B) \cap S\left(y_{0}\right)=\{0\}$.

Given $h_{2} \in \operatorname{null}(B) \cap S\left(y_{0}\right)$, we have $B h_{2}=0$ and $\left\|y_{0}+h_{2}\right\|_{1} \leq\left\|y_{0}\right\|_{1}$. By letting $\left(x^{\prime \prime}, y^{\prime \prime}\right)=\left(x_{0}, y_{0}+h_{2}\right)$, it follows that $z=A x^{\prime \prime}+B y^{\prime \prime}$ and $\left\|y^{\prime \prime}\right\|_{1} \leq\left\|y_{0}\right\|_{1}$. Thus, $h_{2}=0$, otherwise $\left(x^{\prime \prime}, y^{\prime \prime}\right) \neq\left(x_{0}, y_{0}\right)$ will be another minimizer to problem (GDP).

3) Claim: $-A S\left(x_{0}\right) \cap B S\left(y_{0}\right)=\{0\}$.

Given $s \in-A S\left(x_{0}\right) \cap B S\left(y_{0}\right)$, there exist $\bar{x} \in S\left(x_{0}\right)$ and $\bar{y} \in S\left(y_{0}\right)$ to satisfy $-A \bar{x}=B \bar{y}=s,\left\|x_{0}+\bar{x}\right\|_{1} \leq$ $\left\|x_{0}\right\|_{1}$, and $\left\|y_{0}+\bar{y}\right\|_{1} \leq\left\|y_{0}\right\|_{1}$. By letting $\left(x^{\prime \prime \prime}, y^{\prime \prime \prime}\right)=$ $\left(x_{0}+\bar{x}, y_{0}+\bar{y}\right)$, it follows that $z=A x^{\prime \prime \prime}+B y^{\prime \prime \prime}$ and $\left\|y^{\prime \prime \prime}\right\|_{1} \leq\left\|y_{0}\right\|_{1}$, which mean that the point $\left(x^{\prime \prime \prime}, y^{\prime \prime \prime}\right)$ is a feasible point of problem (GDP). On the other hand, since $\left\|x^{\prime \prime \prime}\right\|_{1} \leq\left\|x_{0}\right\|_{1},\left(x^{\prime \prime \prime}, y^{\prime \prime \prime}\right)$ is also an optimal solution. By the fact that the problem (GDP) is assumed to have a unique minimizer $\left(x_{0}, y_{0}\right)$, we conclude that $\left(x^{\prime \prime \prime}, y^{\prime \prime \prime}\right)=\left(x_{0}, y_{0}\right)$; therefore, $\bar{x}=0_{\mathbb{R}^{n_{1}}}, \bar{y}=0_{\mathbb{R}^{n_{2}}}$, and $s=-A \bar{x}=0_{\mathbb{R}^{m}}$.

Conversely, we suppose the point $\left(x_{0}, y_{0}\right)$ satisfies Eq. (III.2). Letting $\left(x^{*}, y^{*}\right)$ be a feasible point of problem (GDP), we show that either $\left\|x^{*}\right\|_{1}>\left\|x_{0}\right\|_{1}$ or $\left(x^{*}, y^{*}\right)=\left(x_{0}, y_{0}\right)$.

Let $h_{1}=x^{*}-x_{0}$ and $h_{2}=y^{*}-y_{0}$. Since $\left(x^{*}, y^{*}\right)$ is feasible in problem (GDP), $z=A x^{*}+B y^{*}=A\left(x_{0}+h_{1}\right)+$ $B\left(y_{0}+h_{2}\right)=z+A h_{1}+B h_{2}$, which implies $-A h_{1}=$ $B h_{2}$. If $\left\|x^{*}\right\|_{1}>\left\|x_{0}\right\|_{1}$, then we are done. So, we may assume $\left\|x^{*}\right\|_{1} \leq\left\|x_{0}\right\|_{1}$, which means $h_{1} \in S\left(x_{0}\right)$. Moreover, $\left\|y^{*}\right\|_{1} \leq\left\|y_{0}\right\|_{1}$ implies $h_{2} \in S\left(y_{0}\right)$. Then, we see that $-A h_{1}=$ $B h_{2} \in-A S\left(x_{0}\right) \cap B S\left(y_{0}\right)=\{0\}$, namely, $h_{1} \in \operatorname{null}(A)$ and $h_{2} \in \operatorname{null}(B)$. Thus, we have $h_{1} \in \operatorname{null}(A) \cap S\left(x_{0}\right)=\{0\}$ and $h_{2} \in \operatorname{null}(B) \cap S\left(y_{0}\right)=\{0\}$, which means $\left(x^{*}, y^{*}\right)=\left(x_{0}, y_{0}\right)$ and we complete the proof.

We know that $S\left(x_{0}\right)$ and $S\left(y_{0}\right)$ are the affine $\ell_{1}$-balls of the points $x_{0}$ and $y_{0}$, respectively. Nevertheless, Eq. (III.2) is the formula, consisting of null spaces of sensing matrices and affine $\ell_{1}$-balls. Indeed, we can relax the affine $\ell_{1}$-ball to be its conical hull, such as $\mathcal{D}\left(\|\cdot\|_{1}, x_{0}\right)=$ cone $\left(S\left(x_{0}\right)\right)$ and $\mathcal{D}\left(\|\cdot\|_{1}, y_{0}\right)=$ cone $\left(S\left(y_{0}\right)\right)$, and attain the following result.

Corollary III.1: The problem (GDP) has a unique minimizer $(\hat{x}, \hat{y})$ that coincides with $\left(x_{0}, y_{0}\right)$ if and only if

$$
\left\{\begin{array}{c}
\operatorname{null}(A) \cap \mathcal{D}\left(\|\cdot\|_{1}, x_{0}\right)=\{0\}, \\
\operatorname{null}(B) \cap \mathcal{D}\left(\|\cdot\|_{1}, y_{0}\right)=\{0\}, \\
-A \mathcal{D}\left(\|\cdot\|_{1}, x_{0}\right) \cap B \mathcal{D}\left(\|\cdot\|_{1}, y_{0}\right)=\{0\} .
\end{array}\right.
$$

We emphasize again that, if $x_{0}$ and $y_{0}$ have the same length, as in Eq. (I.4), then $\mathcal{D}\left(\|\cdot\|_{1}, x_{0}\right)$ and $\mathcal{D}\left(\|\cdot\|_{1}, y_{0}\right)$ will reside 
in the same linear space and their intersection can be geometrically visible, as shown in Fig. 2. Nevertheless, since matrices $A$ and $B$ have arbitrary dimensions in our model, their geometrical interaction cannot be observed simply. Thus, we argue that the derivation of a necessary and sufficient condition via combination of all of the cones is significantly different from the standard problems [1], [2].

\section{Simulations AND Verifications}

We conduct simulations to verify the consistency between Theorem III.1 and GDP.

\section{A. Verification Procedures}

The verification steps for practical sparse signal recovery based on Eq. (III.1) are described as follows.

1) Construct the vectors $x_{0} \in \mathbb{R}^{n_{1}}$ and $y_{0} \in \mathbb{R}^{n_{2}}$ with $k_{1}$ and $k_{2}$ nonzero entries, respectively. The locations of the nonzero entries are selected at random; such a nonzero entry equals \pm 1 with equal probability.

2) Draw two standard normal matrices $A \in \mathbb{R}^{m \times n_{1}}$ and $B$ $\in \mathbb{R}^{m \times n_{2}}$ before capturing the sample $z=A x_{0}+B y_{0}$.

3) Solve problem (GDP) to obtain an optimal solution $\left(\hat{x}_{1}, \hat{y}_{1}\right)$.

4) Declare successful demixing if $\left\|\hat{x}_{1}-x_{0}\right\|_{2} \leq 10^{-5}$. In addition, the verification steps for theoretical recovery based on Theorem III.1 are described as follows.

5) Solve min $\left\|x_{0}+x\right\|_{1}$ subject to $A x=0$ to obtain an optimal point $\hat{x}_{2}$.

6) Solve min $\left\|y_{0}+y\right\|_{1}$ subject to $B y=0$ to obtain an optimal point $\hat{y}_{2}$.

7) Solve min $\left\|x_{0}+x\right\|_{1}$ subject to $A x+B y=0$ and $\| y_{0}+$ $y\left\|_{1} \leq\right\| y_{0} \|_{1}$ to obtain a pair of optimal points $\left(\hat{x}_{3}, \hat{y}_{3}\right)$.

8) Declare success in Theorem III. 1 if $\ell_{2}$-norms of $\hat{x}_{2}, \hat{y}_{2}, \hat{x}_{3}$, and $\hat{y}_{3}$ are all smaller than or equal to $10^{-5}$.

\section{B. Simulation Setting and Results}

In our simulations, $n_{1}$ and $n_{2}$ were the signal dimensions for signals $x_{0}$ and $y_{0}$, respectively. Their sparsities, $k_{1}$ and $k_{2}$, ranged from 1 to $n_{1}$ and 1 to $n_{2}$, respectively.

First, we let $n_{1}=n_{2}=100$ and $k_{1}=k_{2}$. Under this circumstance, the simulation results for both the demixing problems in Eq. (III.1) and Theorem III.1 are illustrated in Fig. 3, where the $x$-axis denotes the sparsity $k$ and the $y$-axis denotes the number $m$ of measurements. We can see that the performance of these two seem to be identical, and we notice that the smaller $k$ is, the easier it is for sparse signal recovery to succeed.

Second, we considered $n_{1} \neq n_{2}$, where $n_{1}=100$ and $n_{2}=160$. Again, $k_{1}$ and $k_{2}$ ranged from 1 to $n_{1}$ and 1 to $n_{2}$, respectively. By additionally considering a varying number of measurements, the visualization of recovery results, unlike Fig. 3, will be multidimensional. So, we chose different numbers of measurements with $10 \leq m \leq 100$ in the simulations to ease observations. The recovery result at each pair of $k_{1}$ and $k_{2}$ for each measurement rate $\left(\frac{m}{n}\right)$ was obtained by averaging from 100 trials. In sum, the simulation results reveal that, if each optimal solution in Steps (5) (7) is zero, then the point, $x_{0}$ and $y_{0}$, satisfies Eq. (III.2), and vice versa. That is to say, we can check if $x_{0}$ and $y_{0}$ satisfy Eq. (III.2) by solving these three optimization problems in Steps (5)-(7).
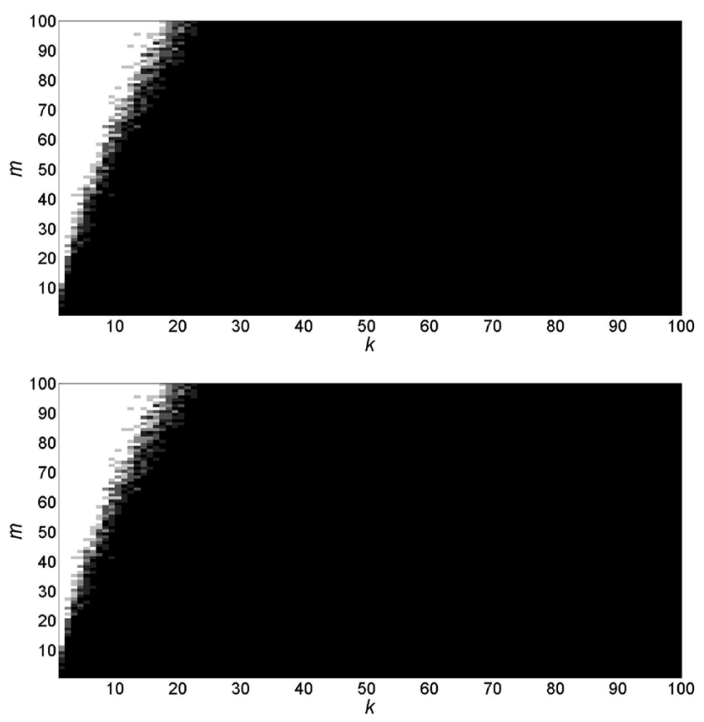

Fig. 3. Phase transitions for demixing problems: [Top] Practical recovery of two sparse vectors based on Eq. (III.1) and [Bottom] theoretic recovery based on Theorem (III.1). In each figure, the heat map indicates the empirical probability of success (Black $=0 \%$; White $=100 \%)$.

\section{Proof of Feasibility of Our Verification}

Now, we show why the above verification is feasible. We say that $\hat{x}_{2}, \hat{y}_{2}, \hat{x}_{3}$, and $\hat{y}_{3}$ obtained from Steps (5)-(7) are all zero vectors if and only if Eq. (III.2) in Theorem III.1 holds. We will validate this claim in the following.

Definition IV.1: Two cones $C$ and $D$ are said to touch if they share a ray but are weakly separable by a hyperplane.

Fact $1[9$, pp. 258-260]

Let $C$ and $D$ be closed and convex cones such that both $C$ and $D \neq\{0\}$. Then,

$$
\mathbb{P}\{\boldsymbol{Q} C \text { touches } D\}=0
$$

where $\boldsymbol{Q}$ is a random rotation.

Lemma IV.1: Steps (5)-(8) constitute a complete verification to (III.2) in Theorem III.1.

Proof: We want to prove that Steps (5)-(8) form a valid verification for Eq. (III.2). First, we assume the point $\left(x_{0}, y_{0}\right)$ satisfies Eq. (III.2).

A1 A1 Claim: $\hat{x}_{2}$ in Step (5) is zero.

Since $\hat{x}_{2}$ is an optimal solution to the problem in Step (5), we have $\left\|x_{0}+\hat{x}_{2}\right\|_{1} \leq\left\|x_{0}\right\|_{1}$, which implies $\hat{x}_{2} \in$ $S\left(x_{0}\right)$, and we have $A \hat{x}_{2}=0$, which is followed by $\hat{x}_{2} \in \operatorname{null}(A)$. That is $\hat{x}_{2} \in \operatorname{null}(A) \cap S\left(x_{0}\right)=\{0\}$; hence, $\hat{x}_{2}=0$.

A2 Claim: $\hat{y}_{2}$ in Step (6) is zero.

The proof is similar to the one in A1.

A3 Claim: $\left(\hat{x}_{3}, \hat{y}_{3}\right)$ in Step (7) is zero.

Since $\left(\hat{x}_{3}, \hat{y}_{3}\right)$ is an optimal solution to the problem in Step (7), we have $\left\|y_{0}+\hat{y}_{3}\right\|_{1} \leq\left\|y_{0}\right\|_{1}$, which means $\hat{y}_{3} \in S\left(y_{0}\right) ;\left\|x_{0}+\hat{x}_{3}\right\|_{1} \leq\left\|x_{0}\right\|_{1}$, which says that $\hat{x}_{3} \in S\left(x_{0}\right)$; and $A \hat{x}_{3}+B \hat{y}_{3}=0$, which implies $-A \hat{x}_{3}=B \hat{y}_{3} \in$ $-A S\left(x_{0}\right) \cap B S\left(y_{0}\right)=\{0\}$. Thus, $\hat{x}_{3} \in \operatorname{null}(A)$ and $\hat{y}_{3} \in \operatorname{null}(B)$, then $\hat{x}_{3} \in \operatorname{null}(A) \cap S\left(x_{0}\right)=\{0\}$ and $\hat{y}_{3} \in \operatorname{null}(B) \cap S\left(y_{0}\right)=\{0\}$, and we come to the conclusion that $\left(\hat{x}_{3}, \hat{y}_{3}\right)=(0,0)$. 


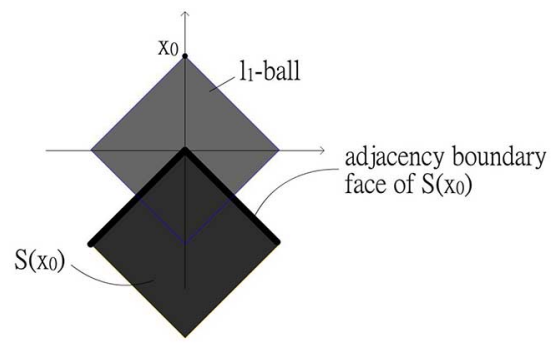

Fig. 4. Adjacency boundary face of $S\left(x_{0}\right)$.

On the other hand, suppose that the optimal solutions $\hat{x}_{2}, \hat{y}_{2}$, and $\left(\hat{x}_{3}, \hat{y}_{3}\right)$ corresponding to the minimization problems in Steps (5), (6), and (7), respectively, are all zeros.

B1 Claim: $\operatorname{null}(A) \cap S\left(x_{0}\right)=\{0\}$.

Given $x^{*} \in \operatorname{null}(A) \cap S\left(x_{0}\right)$, we have $x^{*} \in S\left(x_{0}\right)$, meaning that $\left\|x_{0}+x^{*}\right\|_{1} \leq\left\|x_{0}\right\|_{1}$. Furthermore, we also have $x^{*} \in \operatorname{null}(A)$, which implies that $x^{*}$ is a feasible point of the problem in Step (5). Due to the fact that

$$
\left\|x_{0}\right\|_{1}=\left\|x_{0}+\hat{x}_{2}\right\|_{1} \leq\left\|x_{0}+x\right\|_{1} \forall x \in \operatorname{null}(A),
$$

we have $\left\|x_{0}+x^{*}\right\|_{1} \geq\left\|x_{0}\right\|_{1}$. Thus, $\left\|x_{0}+x^{*}\right\|_{1}=$ $\left\|x_{0}\right\|_{1}$, which means $x^{*}$ belongs to the adjacency boundary face $\partial_{*}\left(S\left(x_{0}\right)\right)$ of $S\left(x_{0}\right)$ at $x_{0}$, where the adjacency boundary face $\partial_{*}(\cdot)=\partial(\cdot) \cap \partial(\operatorname{cone}(\cdot))$ is the intersection of the boundary of itself and the boundary of its conical hull (as shown in Fig. 4). Therefore, $\operatorname{null}(A)$ touches $\mathcal{D}\left(\|\cdot\|_{1}, x_{0}\right)$ or $\operatorname{null}(A) \cap S\left(x_{0}\right)=\{0\}$.

By Fact 1, we may assume that " $\operatorname{null}(A)$ touches $\mathcal{D}(\|$. $\left.\|_{1}, x_{0}\right)$ " never happens. So, we conclude that " $n u l l(A) \cap$ $S\left(x_{0}\right)=\{0\} "$.

B2 Claim: $\operatorname{null}(B) \cap S\left(y_{0}\right)=\{0\}$. The proof is similar to the one in $\mathrm{B} 1$.

B3 Claim: $-A S\left(x_{0}\right) \cap B S\left(y_{0}\right)=\{0\}$.

Given $s^{*} \in-A S\left(x_{0}\right) \cap B S\left(y_{0}\right)$, there exist $x^{*} \in S\left(x_{0}\right)$ and $y^{*} \in S\left(y_{0}\right)$ such that $s^{*}=-A x^{*}=B y^{*}$. Since $y^{*} \in$ $S\left(y_{0}\right)$, we have $\left\|y_{0}+y^{*}\right\|_{1} \leq\left\|y_{0}\right\|_{1}$, together with the fact that $A x^{*}+B y^{*}=0$, the point $\left(x^{*}, y^{*}\right)$ is a feasible point of the problem is Step (7).

Since $\left(\hat{x}_{3}, \hat{y}_{3}\right)=(0,0)$ is an optimal solution to the problem in Step (7), we have

$$
\left\|x_{0}\right\|_{1}=\left\|x_{0}+\hat{x}_{3}\right\|_{1} \leq\left\|x_{0}+x^{*}\right\|_{1} .
$$

Moreover, $x^{*} \in S\left(x_{0}\right)$ means $\left\|x_{0}+x^{*}\right\|_{1} \leq\left\|x_{0}\right\|_{1}$. Thus, $\left\|x_{0}+x^{*}\right\|_{1}=\left\|x_{0}\right\|_{1}$, i.e., $x^{*} \in \partial_{*}\left(S\left(x_{0}\right)\right)$ and $s^{*} \in \partial_{*}\left(-A S\left(x_{0}\right)\right)$. Therefore,

$$
-A S\left(x_{0}\right) \cap B S\left(y_{0}\right) \subseteq \partial_{*}\left(-A S\left(x_{0}\right)\right),
$$

which means $-A \mathcal{D}\left(\|\cdot\|_{1}, x_{0}\right)$ touches $B \mathcal{D}\left(\|\cdot\|_{1}, y_{0}\right)$ or $-A S\left(x_{0}\right) \cap B S\left(y_{0}\right)=\{0\}$. Due to Fact 1 , we may assume that " $-A \mathcal{D}\left(\|\cdot\|_{1}, x_{0}\right)$ touches $B \mathcal{D}\left(\|\cdot\|_{1}, y_{0}\right)$ " never happens. So, we conclude that " $-A S\left(x_{0}\right) \cap B S\left(y_{0}\right)=\{0\} "$.

\section{FUTURE WORK}

We plan to employ Corollary III.1 to estimate the probability of success under some assumptions by the approximate kinematic formula from [1].
Theorem V.1 (Approximate kinematic formula): Fix a tolerance $\eta \in(0,1)$. Let $C$ and $K$ be convex cones in $\mathbb{R}^{n}$, and draw a random orthogonal basis $Q \in \mathbb{R}^{d \times d}$. Then,

$$
\begin{aligned}
& \delta(C)+\delta(K) \leq d-a_{\eta} \sqrt{d} \Rightarrow \mathbb{P}\{C \cap Q K \neq\{0\}\} \leq \eta \\
& \delta(C)+\delta(K) \geq d+a_{\eta} \sqrt{d} \Rightarrow \mathbb{P}\{C \cap Q K \neq\{0\}\} \geq 1-\eta,
\end{aligned}
$$

where $a_{\eta}:=\sqrt{8 \log (4 / \eta)}$ and $\delta$ means the statistical dimension.

Definition V.1 (Statistical dimension): Let $C \subseteq \mathbb{R}^{d}$ be a closed convex cone. Define the Euclidean projection $\Pi_{C}: \mathbb{R}^{d} \rightarrow C$ onto $C$ by

$$
\Pi_{C}(x):=\underset{y \in C}{\arg \min }\|y-x\|^{2} .
$$

The statistical dimension $\delta(C)$ of $C$ is defined as:

$$
\delta(C):=\mathbb{E}_{g}\left[\left\|\Pi_{C}(g)\right\|^{2}\right]
$$

where $g \sim N(0, I)$ is a standard Gaussian vector.

For the generalized demixing model proposed in this paper, we suppose $A \in \mathbb{R}^{m \times n_{1}}$ and $B \in \mathbb{R}^{m \times n_{2}}$ have independent standard normal entries and we let $z=A x_{0}+B y_{0}$. For the compressive sensing demixing, we may assume $m<n_{1}, m<$ $n_{2}$, and both $A$ and $B$ have full rank. Then, we can derive:

$$
\left\{\begin{array}{c}
m \geq \delta\left(\mathcal{D}\left(\|\cdot\|_{1}, x_{0}\right)\right)+a_{\eta_{1}} \sqrt{n_{1}}, \\
m \geq \delta\left(\mathcal{D}\left(\|\cdot\|_{1}, y_{0}\right)\right)+a_{\eta_{2}} \sqrt{n_{2}}, \\
m \geq \delta\left(A \mathcal{D}\left(\|\cdot\|_{1}, x_{0}\right)\right)+\delta\left(B \mathcal{D}\left(\|\cdot\|_{1}, y_{0}\right)\right)+a_{\eta_{3}} \sqrt{m}
\end{array}\right.
$$

which implies

$$
\left\{\begin{array}{l}
\mathbb{P}\left\{\operatorname{null}(A) \cap \mathcal{D}\left(\|\cdot\|_{1}, x_{0}\right)=\{0\}\right\} \geq 1-\eta_{1}, \\
\mathbb{P}\left\{\operatorname{null}(B) \cap \mathcal{D}\left(\|\cdot\|_{1}, y_{0}\right)=\{0\}\right\} \geq 1-\eta_{2}, \\
\mathbb{P}\left\{-A \mathcal{D}\left(\|\cdot\|_{1}, x_{0}\right) \cap B \mathcal{D}\left(\|\cdot\|_{1}, y_{0}\right)=\{0\}\right\} \geq 1-\eta_{3} .
\end{array}\right.
$$

On the other hand, we also have

$$
\left\{\begin{array}{c}
m \leq \delta\left(\mathcal{D}\left(\|\cdot\|_{1}, x_{0}\right)\right)-a_{\eta_{1}} \sqrt{n_{1}} \\
m \leq \delta\left(\mathcal{D}\left(\|\cdot\|_{1}, y_{0}\right)\right)-a_{\eta_{2}} \sqrt{n_{2}} \\
m \leq \delta\left(A \mathcal{D}\left(\|\cdot\|_{1}, x_{0}\right)\right)+\delta\left(B \mathcal{D}\left(\|\cdot\|_{1}, y_{0}\right)\right)-a_{\eta_{3}} \sqrt{m}
\end{array}\right.
$$

which implies

$$
\left\{\begin{array}{c}
\mathbb{P}\left\{\operatorname{null}(A) \cap \mathcal{D}\left(\|\cdot\|_{1}, x_{0}\right)=\{0\}\right\} \leq \eta_{1}, \\
\mathbb{P}\left\{\operatorname{null}(B) \cap \mathcal{D}\left(\|\cdot\|_{1}, y_{0}\right)=\{0\}\right\} \leq \eta_{2}, \\
\mathbb{P}\left\{-A \mathcal{D}\left(\|\cdot\|_{1}, x_{0}\right) \cap B \mathcal{D}\left(\|\cdot\|_{1}, y_{0}\right)=\{0\}\right\} \leq \eta_{3} .
\end{array}\right.
$$

Apparently, if the number $m$ of measurements is large enough, then successful sparse recovery can be achieved. On the other hand, failed recovery is possible due to an insufficient number of measurements. Nevertheless, if we want to realize the above derived results, computation of the statistical dimensions of $A \mathcal{D}\left(\|\cdot\|_{1}, x_{0}\right)$ and $B \mathcal{D}\left(\|\cdot\|_{1}, y_{0}\right)$, as indicated in Eqs. (V.1) and (V.2), will be an unavoidable difficulty.

\section{CONCLUSION}

Our major contribution in this paper is to derive the necessary and sufficient condition for a successful generalized demixing problem. There is an issue worth mentioning, i.e., Amelunxen et al. have evaluated an upper bound and a lower bound of the probability of successful recovery for the demixing problem (DP). The reason we did not do that is due to the known unavoidable difficulty raised by the generalized model (GDP problem), that is, "How to compute the statistical dimension of a descent cone operated by a linear operator?" We believe that, if this open problem can be solved, we will complete the generalized demixing problem with Gaussian random measurements. 


\section{REFERENCES}

[1] D. Amelunxen, M. Lotz, M. B. McCoy, and J. A. Tropp, "Living on the edge: A geometric theory of phase transitions in convex optimization," arXiv preprint arXiv:1303.6672, 2013.

[2] M. B. McCoy and J. A. Tropp., "The achievable performance of convex demixing," arXiv preprint arXiv:1309.7478, 2013.

[3] V. Chandrasekaran, B. Recht, P. A. Parrilo, and A. S. Willsky, "The convex geometry of linear inverse problems," Found. Comput. Math., vol. 12, no. 6, pp. 805-849, 2012.

[4] S. S. Chen, D. L. Donoho, and M. A. Saunders, "Atomic decomposition by basis pursuit," SIAM Rev., vol. 43, no. 1, pp. 129-159, 2001.

[5] D. L. Donoho, "Compressed sensing," IEEE Trans. Information Theory, vol. 52, no. 4, pp. 1289-1306, 2006.
[6] J.-B. Hiriart-Urruty and C. Lemarchal, "Convex analysis and minimization algorithms," in I, volume 305 of Grundlehren der Mathematischen Wissenschaften [Fundamental Principles of Mathematical Sciences]. Berlin, Germany: Springer-Verlag, 1993, Fundamentals.

[7] M. Rudelson and R. Vershynin, "On sparse reconstruction from Fourier and Gaussian measurements," Commun. Pure Appl. Math., vol. 61, no. 8, pp. 1025-1045, 2008

[8] C. Studer and R. G. Baraniuk, "Stable restoration and separation of approximately sparse signals," Appl. Comput. Harmon. Anal., vol. 37, pp. $12-35,2014$.

[9] R. Schneider and W. Weil, Stochastic and Integral Geometry, ser. Series in Statistics: Probability and its Applications. Springer. Berlin, Germany: Springer, 2008

[10] S. S. Chen, D. L. Donoho, and M. A. Saunders, "Reprinted from SIAM,” J. Sci. Comput., vol. 20, no. 1, pp. 33-61, 1998. 